\title{
The evolution of novel biotic interactions at ecological margins in response to climate change involves alleles from across the geographical range of the UK Brown Argus butterfly
}

\section{Running title}

6 Rapid adaptation in the UK Brown Argus butterfly uses widespread genome variation

Maaike de Jong ${ }^{1,5 *}$, Alexandra Jansen van Rensburg ${ }^{1,4 *}$, Samuel Whiteford ${ }^{2}$, Carl J. Yung ${ }^{2}$, 10 Mark Beaumont $^{1}$, Chris Jiggins ${ }^{3}$, Jon Bridle ${ }^{1,4+}$

*these authors contributed equally to this publication

${ }^{1}$ School of Biological Sciences, University of Bristol, BS8 1UD, UK

${ }^{3}$ Department of Genetics, University of Cambridge, UK

${ }^{4}$ Current address: Department of Genetics, Evolution and Environment, University College London, WC1E 4HE, UK.

${ }^{5}$ Current address: Netherlands eScience Center, 1098 XG Amsterdam, The Netherlands.

+To whom correspondence should be addressed: j.bridle@ucl.ac.uk 


\section{Abstract (222 words)}

Understanding the rate and extent to which populations can adapt to novel environments at

their ecological margins is fundamental to predicting the persistence of biological

communities during ongoing and rapid global change. Recent range expansion in response to climate change in the UK butterfly Aricia agestis is associated with the evolution of novel interactions with a larval food plant, and the loss of its ability to use its ancestral larval host species. Using ddRAD analysis of 61210 variable SNPs from 261 females from throughout the UK range of this species, we identify genomic regions at multiple chromosomes that are associated with these evolutionary responses, and their association with demographic history and ecological variation. Gene flow appears widespread throughout the range, despite the apparently fragmented nature of the habitats used by this species. Patterns of haplotype variation between selected and neutral genomic regions suggest that evolution associated with climate adaptation is polygenic, resulting from the independent spread of existing alleles throughout the established range of this species, rather than the colonisation of pre-adapted genotypes from coastal populations. These data suggest that rapid responses to climate change do not depend on the availability of pre-adapted genotypes. Instead, the evolution of novel forms of biotic interaction in Aricia agestis has occurred during range expansion, through the assembly of novel genotypes from alleles from multiple localities. 


\section{Introduction}

Predicting population and community persistence in the face of a changing and more variable climate remains an urgent priority for biologists (Bridle \& van Rensburg 2020). A critical unknown is when and to what extent evolutionary responses will buffer the effects of climate change on ecological communities, or will allow species to shift their ranges to track changes in suitable climate (Hoffmann \& Sgrò 2011; Angert et al. 2020). Of particular interest is how specialist interactions between species (e.g. parasites and hosts, herbivores and food plants) will limit the availability of suitable habitat, so preventing range shifts (Chen et al. 2011), and how these interactions will change as species encounter novel environmental regimes (Nadeau et al. 2017; Hoffmann \& Bridle 2021), O’Brien et al. in press).

Population genetic models of adaptation at ecological margins predict that steep and patchy ecological gradients prevent species from tracking changing climate, leading to local (and eventually global) extinction (Polechová \& Barton 2015; Bridle et al. 2019). Other models of evolution at range margins predict that adaptive shifts are more likely if historic populations have been exposed to variable environments which maintain genetic variation across the species geographical range (Kopp \& Matuszewski 2014). In support of this, empirical studies suggest that, although many generalist species have shifted their distributions to track available habitat, almost $75 \%$ species with specialist biotic interactions have declined or failed to shift their ranges to match suitable climate (Parmesan 2006; Hill et al. 2011).

A key question is whether rapid evolution at ecological margins depends on genotypes already present somewhere in a species' geographical range. For example, under global climate warming, alleles at the equatorial part of a species' range may support adaptation at 
poleward margins, provided gene flow is sufficiently widespread. Such widespread gene flow among marine populations is likely to explain repeated radiations of sticklebacks into freshwater lakes from their marine ancestors (Jones et al. 2012). If such pre-existing (“standing”) allelic variation is necessary for rapid evolution, population persistence may depend on maintaining large populations throughout the species' geographical range, or on translocations from appropriate environments if dispersal among populations is limited (Bridle et al. 2009; Hoffmann \& Sgrò 2018). By contrast, if rapid evolution depends on novel mutations, then a population's current size is more relevant in predicting its adaptive potential, rather than its connections with other populations, or its historical size.

Many Lepidoptera species are associated with particular host plant species, particularly at the larval stage, due to plant defence against herbivory, and the particular microclimates that host plants offer as oviposition sites (Jaenike 1990; Stewart et al. 2021). Such specialist biotic interactions slow or prevent range expansion into areas that may be climatically suitable, but where the preferred host plant is rare. In the UK, more than $90 \%$ of Lepidoptera species that are habitat specialists have contracted their ranges (Warren et al. 2001). Limits to habitat availability caused by specialist host plant interactions are also associated with lags in climate responses (Chen et al. 2011), with habitat availability explaining $25 \%$ of the variation in range expansion rates, even accounting for other factors (Platts et al. 2019). Such effects of habitat availability suggest that range shifts in specialists in response to climate change depend on the evolution of novel biotic interactions at ecological margins. Habitat specialists that have tracked changing climates therefore provide an exceptional opportunity to understand the evolutionary responses demanded by ecological gradients made locally steep by particular biotic interactions (Bridle et al. 2019; O'Brien et al. in press). 
The Brown Argus butterfly, Aricia agestis (Polyommatinae: Lycaenidae), is a habitat

(Asher et al. 2001) in response to climate change (Thomas et al. 2001; Bodsworth 2002;

Pateman et al. 2012). In mainland Europe, annual plants from the family Geraniaceae

(Geranium and Erodium) are its predominant larval hosts (Tolman 1997; Asher et al. 2001).

For most of the $20^{\text {th }}$ century in the UK, however A. agestis only used Geraniaceae as a host

103 grows only on chalk or limestone soils, and its perennial growth form probably provides a predictable larval microclimate and food supply for $A$. agestis, regardless of annual temperature variation (Stewart et al. in press). By contrast, Geranium annuals are 4-7 times more sparsely distributed across the landscape, and are more affected by seasonal climate,

107 performing well during wet springs and warm summers, but becoming rare and lower quality

108 when springs are dry and summers are wet (Pateman et al. 2012; Stewart et al. 2021).

110 These data suggest that for most of the $20^{\text {th }}$ century, A. agestis could persist solely in the UK

111 on Geraniaceae host plants where south-facing sand dunes provided locally elevated

112 microclimates for rapid larval growth. However, increasing summer temperatures since the

113 1990s have made non-coastal Geranium populations available for sustained occupation by $A$.

114 agestis for the first time, leading to rapid range expansion northward, into areas where

115 Rockrose host plants are rare (Pateman et al. 2012; Figure 1). 
117 Studies of female oviposition preference and genotyping at AFLP markers demonstrate that

118 range expansion into higher latitude Geranium sites has been associated with evolutionary

119 change (Buckley et al. 2012). Newly-colonised areas show an increased preference of

120 mothers to oviposit on the widespread G. molle (Geranium) (Thomas et al. 2001; Bridle et al.

121 2014), as well as reduced variation in female oviposition preference, increased dispersal

122 ability, and less consistent preference for the locally most common host plant species (Bridle et al. 2014). In addition, field transplants of individual females onto natural host plants

126 dominated (newly-colonised) sites north of the range will only oviposit on Geranium plants

127 (Buckley \& Bridle 2014). This suggests that range expansion driven by climate adaptation in

A. agestis has been associated with a narrowing of oviposition preference (albeit to exploit a plants in the newer parts of its range.

132 The example of the UK Brown Argus suggests that climate-driven range shift by a habitat specialist has required an evolutionary change in species' interactions. In this case, evolution

134 of host plant has effectively smoothed a steep and patchy ecological gradient at the species'

135 poleward margin, allowing access to habitats that have recently become climatically suitable,

136 even though they lack the host plants typically used by long-established populations (O'Brien

137 et al. in press). Such a system represents an exceptional opportunity to understand the

138 ecology and genetics of rapid adaptation, and to assess the likelihood and likely context of

139 such adaptation in other species and circumstances, particular for species that depend on

140 particular interactions with other species. In A. agestis, a key question is whether its 
expansion involved the colonisation of novel areas by females from coastal UK populations

142 that already used only Geraniaceae food plants. Alternatively, did the observed shift in

143 species' interactions involve the creation of novel genotypes in situ, either from new

144 mutations arising locally, or from selection on standing genetic variation already found in

145 southern UK populations that are able to use both Rockrose and G. molle (Buckley and

146 Bridle, 2014).

148 In this paper, we use genome-wide SNP markers to assess the distribution of genetic variation

149 across the UK, and to test the genetic basis of adaptation at the newly colonised sites.

150 Specifically, we: (1) test for reduced genomic variation associated with range expansion,

151 suggesting selective sweeps at the range margin for specialisation on Geranium host plants; 2)

152 identify regions of the genome under selection, and their genomic distribution and likely

153 function; and 3) determine whether evolution during range expansion has occurred through

154 colonisation of existing genotypes that use only Geranium host plants, or through the

155 assembly of novel genotypes from alleles sourced from throughout the geographical range.

156

157 Materials and Methods

159 Study system and sample collection

160 Nine Aricia agestis populations (276 individuals, 19-45 sampled per site) were sampled in the

161 summers of 2013 and 2014 across their latitudinal distribution in the UK. Populations were

162 chosen to include long established (present since 1970-1982) and newly colonised sites (since

163 1995-1997; Thomas et al. 2001), and sites were classified as either dominated by 
164 Geraniaceae (which includes G. molle, G. dissectum, and Erodium cicutarium) or Cistaceae

(Rockrose Helianthemum nummularium; Table 1).

Generating genome-wide markers for population genetics

168 DNA was extracted from the head and half of the thorax each of 276 individuals using a

169 Qiagen DNeasy Blood and Tissue kit. DNA was eluted in EB buffer and quantified using

Qubit 2.0 fluorometer with the DNA BR assay kit (Life Technologies).

172 Genome wide markers were generated using a modified double digest restriction associated

173 DNA (ddRAD) protocol (Peterson et al. 2012). Briefly, genomic DNA of each individual was

174 digested with $P s t I$ and EcoRI restriction enzymes. In total 276 individuals from nine

175 populations were sequenced across six ddRAD libraries. Individuals from a single population

176 were sequenced in at least four different libraries to avoid confounding population structure

177 with differences in library preparation and sequencing between libraries. Each library

178 comprised 48 individuals uniquely identified using a 6-bp DNA barcode. Libraries were

179 sequenced on an Illumina HiSeq 2000 instrument to generate 150-bp paired-end sequences

180 from insert sizes of 300-450 bp.

\section{Bioinformatic analysis}

183 Raw data were demultiplexed based on individual barcodes using ipyRAD v. 0.7.28 (Eaton

184 2014) and adapters were removed using Trimmomatic v. 0.36 (Bolger et al. 2014). A long-

185 read based reference genome has been assembled by the Sanger Institute and is available

186 under accession number GCA_905147365.1 from the National Center for Biotechnology

187 Information (NCBI; www.ncbi.nlm.nih.gov). To call variants we first mapped the 

as a VCF and filtered before downstream analyses using VCFtools v.0.017 (Danecek et al.

193 2011), BCFtools v. 1.8, and PLINK 2.0 (Purcell et al. 2007).

The following filters were applied: 1) We retained only variants called with a genotype was applied to reduce spurious heterozygote calls. 3) A maximum mean depth of the mean

198 plus twice the standard deviation (646x) was applied to remove duplicate loci. 4) Kinship coefficients were estimated between individuals within each population using the KING method (Manichaikul et al. 2010). Individuals were removed to exclude any second order or higher relatives (phi $>0.05$ ). 5) Individuals with a genotyping rate of less than $60 \%$ were removed. 6) To include loci that were evenly genotyped across all populations, we excluded

203 loci genotyped in less than $50 \%$ of individuals within each population. 7) We allowed a 204 global minimum minor allele frequency (MAF) of 1\%. The final dataset comprised 251 205 individuals genotyped at 61210 variants.

207 (1) Changes in genomic variation associated with range expansion

Population structure and genetic variation associated with range expansion

210 We assessed population structure and genetic diversity across the sampled range using three 211 complementary methods. First, we visually examined the extent of genetic differentiation 
between populations using a Principal Component Analysis (PCA) using the R package approximate the log-marginal likelihood of the data. This approach is attractive for large

216 genomic datasets because it is very rapid compared to a traditional Bayesian approach implemented in Structure (Pritchard et al. 2000). We estimated ancestry proportions for up to

21810 clusters $(\mathrm{K}=2-10)$ using a simple prior on the model. Thirdly, we estimated individual coancestry based on a pairwise comparison between samples using a Markov chain Monte Carlo (MCMC) coalescence model implemented in fineRADstructure (Malinsky et al. 2018).

221 fineRADstructure estimates coancestry between individuals by comparing haplotypes across

222 all individuals to estimate the nearest neighbour for each locus. Coancestry is divided equally between all individuals with the same haplotype, or between individuals that are the nearest neighbour of a rare haplotype. In this way rare haplotypes and their nearest neighbours receive a higher coancestry weighting than more prevalent haplotypes. Given that rare mutations are on average expected to be of more recent origin than haplotypes that occur at 227 higher frequencies in the population, fineRADstructure is able to estimate recent coancestry 228 in the dataset. No population prior was specified for the analysis. We ran the analysis with a 229 burn-in of 100'000 iterations, followed by 100'000 MCMC steps. RADpainter (Malinsky et 230 al. 2018) was used to infer the coancestry matrix and assign individuals to populations using 231 default parameters.

233 To determine whether genetic divergence between populations increases on average with 234 geographic distance, we estimated population pairwise F $_{\text {ST }}$ (Nei 1973) from a subset of 235 unlinked loci using the R package adegenet (Jombart et al. 2008). We estimated Pearson's 
correlation coefficient between genetic distance and log transformed geographic distance, tested with a Mantel test in the vegan package (Oksanen et al. 2015) in R.

Testing for changes in host plant use at the range edge

241 We tested for genome-wide signals of specialisation on the most prevalent host plant at each

242 site by determining how much genetic variance can be explained by 1) host plant prevalence

243 and 2) site colonisation history. The initial model (Basic Model) determined how much of the

244 genetic variance could be explained by all the variables combined using a redundancy

245 analysis (RDA) as implemented in the vegan package (Oksanen et al. 2015) in R. The full

246 Basic Model was GeneticData [MAF matrix] Latitude + Longitude + Host Plant +

247 Colonisation History. Next, we determined the best model to explain variance in the genetic

248 data by removing one non-significant variable at a time based on an automatic permutation of

249 the model and selecting the best variables in each case based on Akaike's Information

250 Criterion (AIC). This was implemented using the ordistep function from vegan in R. We then 251 used two partial RDA analyses to estimate the variance explained by Host Plant or

252 Colonisation History when Latitude and Longitude are kept constant.

254 Previous data on UK Aricia agestis suggests that populations expanding at the range margin 255 lost genetic diversity due to the spread of genotypes laying only on Geraniaceae hosts rather 256 than on Geraniaceae and rockrose hosts (Bridle et al. 2014; Buckley \& Bridle 2014). We 257 tested for a signal of a genome-wide reduction in genetic variation associated with range 258 expansion by comparing gene diversity and mean genome-wide nucleotide diversity between 259 sites dominated by the different host plants or with different colonisation histories. Nucleotide 
diversity was calculated across $1 \mathrm{~kb}$ windows within each population using VCFtools v.0.1.14.

Expected heterozygosity was calculated using the basic.stats function in the R package

hierfstat (Goudet 2005). We tested whether these estimates differed significantly between

sites using a Kruskal-Wallis rank sum test implemented in R. We tested the robustness of these pairwise comparisons using two methods: 1) a jackknife approach, where we repeated the analysis while removing each population in turn, and 2) we randomised the colonisation history and host plant variables and repeated the Kruskal-Wallis rank sum tests.

(2) Identifying genomic regions under selection and their geographical distribution

Identifying genomic regions under selection across the UK

272 We used the FST outlier approach implemented in BayeScan v2.1 (Foll \& Gaggiotti 2008) to

273 identify adaptive genotypes associated with 1) different host plants and 2) site colonisation

274 history. This model decomposes $\mathrm{F}_{\mathrm{ST}}$ between populations into a population (beta) and locus

275 (alpha) component. A locus is deemed to be under selection if the alpha component is needed to explain the diversity at that locus. BayeScan implements a reverse-jump MCMC to explore

277 the parameter space with and without the alpha parameter included, and therefore estimates a 278 posterior probability associated with each model. To identify and exclude any loci associated 279 with a single population, we repeated the analysis with each population removed in turn. Loci 280 that were identified with a false discovery rate of 0.05 in the full analysis and at least two 281 thirds of the jackknife analyses were included in the final set of outlier loci. 
284 We annotated the outlier loci with snpEff (Cingolani et al. 2012) using a database built from

285 the reference genome and annotation available on NCBI (GCA_905147365.1). We used the

286 "closest" function to identify the closest gene to each outlier locus. The gene name and

287 biological process was obtained by searching the UniProtKB database (The UniProt

288 Consortium 2021) for the protein identified in each case.

(3) Determining the geographical and colonisation history of evolutionary responses

Haplotype networks of outlier loci associated with host plant variation

293 To determine if alleles associated with adaptation to different host plants spread

294 independently, or as genotypes during population expansion, we constructed haplotype

295 networks for each of the loci by identifying and phasing all outlier ddRAD tags. We subset

296 the mapped reads (bam files) by extracting the outlier variants flanked by $300 \mathrm{bp}$ on either side

297 to include all variants within the RAD tag. We kept only sequences with at least two variant

298 sites. We phased each locus with WhatsHap (Patterson et al. 2015), which uses sequence data

299 from each individual's bam files to inform phasing. All unphased sites were removed. We

300 extracted the haplotype sequences in fasta format and constructed haplotype networks for

301 each locus using the Pegas package (Paradis 2010) in R.

To compare the spread of adaptive versus neutral loci across the landscape we used the same

304 approach to extract a random set of 20 neutral sequences. After removing sequences with less

305 than two variant sites we reconstructed haplotype networks for the 11 remaining sequences. 
The evolution of $A$. agestis to use only Geraniaceae at newly-colonised sites could have occurred through the arrival of pre-adapted genotypes that already specialised on these host plants, or through evolution in situ at the range edge. The established north-eastern population (HOD) is dominated by Geraniaceae and its geographic proximity to newly-colonised sites makes it a potential local source for such pre-adapted genotypes. To test this idea, and to

313 determine the most likely origin of colonists at the new sites, we constructed six demographic models scenarios using the coalescent simulator fastSimCoal2 (Excoffier et al. 2013). We compared three models of demographic history with two different migration scenarios applied colonisation of the new sites (Figure 2). We applied two migration scenarios to each model for a total of six models: a) a full migration matrix of asymmetric gene flow, and b) a by our tests for selection and excluded the Z-chromosome from our analysis.

Missingness is a characteristic of reduced representation libraries because loci sequenced in different libraries don't overlap exactly. However, if we minimise the missingness in the dataset, too few loci remain to accurately estimate the site frequency spectrum. At the same time, including sites with a high proportion of missingness can bias the estimated site frequency spectrum. Therefore, to increase the number of loci in the final dataset, we used resampled data within populations to reduce missingness. This approach resamples loci that have a user-specified minimum number of genotypes across all samples. In this way, 330 missingness within an individual is circumvented and a full genotype matrix can be created. 331 Our final dataset comprised 9735 SNPs. Scripts to downsample the data and construct the 
minor allele frequency spectrum were obtained from Vitor Sousa, University of Lisbon

The effective population size was fixed for "South" in the model so that all parameters could be estimated relative to this value. The effective population size $\left(N_{e}\right)$ was calculated from the mutation rate $(\mu)$ and nucleotide diversity $(\pi)$. Nucleotide diversity was calculated across all variant and invariant sites in windows of $1 \mathrm{~kb}$ using vcftools (--window-pi function; $\pi=0.001$ ). We assume a mutation rate of $2.9 \times 10^{-9}$ per base per haploid genome per generation based on 340 the only direct estimate of Lepidoptera mutation rates (H. melpomone, Keightley et al. 2014).

341 The median effective population size was calculated as $N_{e}=(\pi / 4 \mu$ ); South $=86207$ (range 898342 712069). For parameter estimation we assume a generation time of 0.5 years, because $A$. 343 agestis in Britain are bivoltine.

345 Given the uncertainty associated with these parameters, and the small site frequency spectrum 346 compared with the number of parameters estimated (9-15), we expect wide and overlapping 347 confidence intervals and uncertainty in the parameter estimates. This meant that we used the 348 test to determine the relative likelihood of the different demographic models, rather than the 349 absolute values of the estimated parameters. We ran 100 independent simulations of each 350 model in fastSimCoal2. Each run comprised 100000 coalescent simulations and 40 351 expectation maximisation cycles. All parameters and priors are documented in Table S4. We 352 evaluated the model fit based on the lowest log likelihood and Akaike's information criterion 353 (AIC). To compare the models directly we rescaled AIC as the difference between the lowest 354 AIC and the AIC for each competing models. This is shown in the results as $\triangle \mathrm{AIC}$, where the 
355 best model has a $\triangle \mathrm{AIC}$ of 0 (Table 4). The point estimates of each demographic parameter for

356 the best supported model were obtained from the run with the highest composite maximum

357 likelihood score.

359 Confidence intervals (CI) were estimated for the parameters by simulating 100 site frequency

360 spectra using the maximum likelihood point estimates for the best run. Parameter estimates

361 were re-estimated using 100 independent simulations of the model for each of the simulated

362 site frequency spectra The lower and upper CI bounds were obtained from the lowest and

363 highest composite maximum likelihood estimates obtained from the 100 iterations.

\section{Results}

367 After applying filters, the final datasets comprised 251 individuals from nine populations $368(\mathrm{n}=15-38 ;$ Table1 $)$ genotyped at 61210 variants.

\section{0 (1) Changes in genomic variation associated with range expansion}

Population structure is associated with latitude

373 Our results suggest that $A$. agestis populations are largely structured latitudinally, following

374 the likely colonisation route northwards, with the exception of FOR (see below). Overall gene

375 flow was high $\left(\mathrm{F}_{\mathrm{ST}}=0.031\right.$ between all sites; Table 1, Table $\left.\mathrm{S} 1\right)$, but genetic distance increased

376 significantly with geographic distance (Mantel's $r=0.78, p=0.001)$. Similarly, genetic

377 divergence was low $\left(\mathrm{F}_{\mathrm{ST}}=0.026\right)$ but significant between established and new sites (Mantel's $\mathrm{r}$ 
$378=0.45, p=0.04)$, with estimates similar to previous results based on 409 AFLP markers

379 (FST=0.025; Buckley et al. 2012). By contrast, genetic divergence was not significant between

380 sites dominated by different host plants $\left(\mathrm{F}_{\mathrm{ST}}=0.018\right.$; Mantel's $\left.\mathrm{r}=0.29, p=0.07\right)$.

382 Our PCA analysis showed that the most genetic variance was explained by the divergence

383 between northern and southern populations (PCA1 $=3.7 \%$; Figure 1), with FOR more closely

384 related to the southern populations than the other northern populations, and HOD more

385 differentiated than the others. Similarly, fastStructure results supported two genetic clusters

$386(\mathrm{~K}=2)$ that also correspond to the northern and southern populations (Figure 1). Both analyses

387 showed that HOD (the only established population where Geraniaceae is the most prevalent

388 host plant) is differentiated from the rest of the UK sites. In the PCA, the divergence between

389 HOD and the rest of the populations explains $1.3 \%$ of the total genomic variance (PC2).

390 Results from fastStructure also reveal that HOD and BAR (a newly established site) are

391 genetically intermediate to the northern and southern clusters (Figure 1).

393 Recent coancestry as estimated with fineRADstructure revealed a more complex relationship

394 between populations. Two well-supported groups were recovered that correspond to the

395 northern and southern populations. However, HOD clustered with the three established

396 southern populations (Figure 3). Substructure within the northern cluster supported

397 differentiation between the newly established Geraniaceae dominated sites and the two sites

398 where Cistaceae are the most prevalent. The Cistaceae dominated sites (FOR and BAR) are

399 geographically well separated, and the Geraniaceae sites (BRO, WIS, MOF) are found

400 between them. This suggests that the new sites were colonised from the established Cistaceae

401 sites, rather than the established Geraniaceae site to the east (HOD). There is also evidence of 
colonisation through infilling from neighbouring populations, with at least five individuals in

Geraniaceae haplotypes (Figure 3).

Genomic variation is slightly reduced in newly-colonised regions

407 Our results show that there is genome-wide differentiation between new and established sites, 408 and between Geraniaceae and Cistaceae dominated sites, but that the variance in the data cannot be significantly explained by either of these variables, independent of spatial variables (Table 2). The Basic Model explained a large and significant proportion of variance in the

411 genomic data (Basic Model: 66\%, $\mathrm{p}=0.001$ ). The best model retained Latitude and

412 Colonisation History as significant variables. Using partial redundancy analyses we found a 413 large proportion of constrained variance explained by HostPlant use (10\%) and Colonisation 414 History (14\%), but neither were significant in the Basic Model.

416 Genetic diversity was marginally but significantly lower in newly colonised sites when 417 compared with established sites (Table 1, Figure S1; median Hs, $\pi$ : new $=0.12,0.00074$; 418 established $=0.13,0.00077)$, and in Geraniaceae dominated sites compared with Cistaceae 419 sites (median Hs, $\pi$ : Geraniaceae $=0.12,0.00074$; Cistaceae $=0.13,0.00077)$. For 420 comparisons based on nucleotide diversity the test remained highly significant $(p<0.001)$ 421 except with the removal of MOF or FOR. Randomisation of the host plant or colonisation 422 history variable resulted in non-significant differences. Allelic diversity was significantly 423 different in all cases $(p<2.2 \mathrm{e}-16)$, but was still significant when the variables were 424 randomised $(p=0.026)$. 
(2) Identifying genomic regions under selection and their distribution

We identified 12 loci (137 SNPs; $0.22 \%$ of total variants) associated with host plant preference, and 19 loci (239 SNPs; 0.39\%) associated with colonisation history. Two loci each on the Z-chromosome and Chromosome 9 were identified as outliers in both "colonization history" and "host plant use" datasets (Table S2). Of the 25 candidate loci that could be assigned an annotation, 21 were located in the intron of a gene, and the remaining

History comparison.

(3) Determining the geographical and colonisation history of evolutionary responses only a few loci (Van Belleghem et al. 2018; Kautt et al. 2020). This suggests that selection on variation in Host Plant preference acts across many loci with high levels of recombination 
450 haplotype currently found in new Geraniaceae sites was derived from the established coastal

451 Geraniaceae site (HOD). Instead, we found that the Geraniaceae-preferring haplotypes in

452 newly-colonised areas were similar to common haplotypes that occur in both the South and

453 HOD, or only in the South (Table S3). In addition, haplotype networks of neutral loci were

454 indistinguishable from the adaptive loci, which suggests that the adaptive and neutral alleles

455 spread to the new Geraniaceae populations from both the established populations in the South

456 (that can use both Geranium and rockrose) and from coastal populations using Geraniaceae,

457 across sufficient numbers of generations for recombination to occur between them.

Reconstructing the colonisation history using a coalescent approach

460 The best-supported colonisation history model (Model3a) specified that New sites were

461 colonised by an admixture of the established North-Eastern population (HOD) and the

462 established populations in the South (Table 3; Fig 2). Point estimates of the model parameters

463 suggests a slightly higher proportion of ancestry from the South (56\%) than from HOD (44\%)

464 (Table 4), although this should be interpreted with caution based on the wide confidence

465 intervals surrounding our estimates.

466

467 


\section{Discussion}

\section{Rapid adaptation occurs by formation of genotypes from alleles across the range}

472 The population genomic analyses presented here suggest that the rapid evolution of host plant

473 use at the expanding range edge in A. agestis is associated with selection on genomic

474 variation found across the species' range and facilitated by high levels of gene flow between

475 populations. Our analyses of haplotype data (Figure 5) and fastSimCoal2 simulations best

476 support a scenario where climate adaptation occurred through evolution in situ during range

477 expansion, rather than through colonisation by pre-adapted genotypes from coastal

478 populations that already specialise on the new host plant. This finding is supported by the fact

479 we find little evidence for a reduction in genome-wide genetic variance at newly-colonised

480 sites, despite evidence that female oviposition preference has narrowed in the new habitats

481 (Buckley \& Bridle 2014). Buckley and Bridle (2014) also provide evidence for reduced

482 fitness of long-established Geraniaceae-using populations on the Norfolk coast, when

483 transplanted to Geranium sites in Lincolnshire, suggesting that established forms of

484 Geraniaceae use may involve different traits to those associated with climate-driven range

485 expansion.

487 FineRADstructure plots, and the lack of structure in the haplotype networks both in the

488 adaptive and neutral loci, suggest ongoing gene flow from source habitats, or subsequent gene

489 flow following an initial bottleneck associated with colonisation. Range expansion in $A$.

490 agestis seems to occur by infilling of new habitats rather than a mass northward expansion

491 per se, and with historical and ongoing high connectivity between neighbouring (source)

492 populations. The lack of a strong signal in population size associated with range expansion 
could also be explained by a polygenic genomic architecture associated with the host-plant shift, meaning that selective sweeps during adaptation to novel conditions have had little effect on effective population size (see below).

\section{Shifts in host plant use associated with climate-driven range expansion}

Although $A$. agestis females from the core range typically prefer Rockrose in field host choice experiments (Bridle et al 2014), they can also oviposit on Geraniaceae. However, the expansion into northern England has been associated with the loss of Rockrose use, certainly for Lincolnshire populations (Buckley and Bridle, 2014). Presumably therefore, some cost to maintaining preference for both host plants is responsible for the loss of rockrose laying preference in habitats where only Geraniaceae is present. Geographic variation in host plant choice has been found in several polyphagous butterfly species (e.g. Hanski et al. 2002; Nygren et al. 2006; Stålhandske et al. 2016), and these are driven by trade-offs such as differences in the host plant defence chemicals, host plant morphology and life history (e.g. phenology), or the regional and local abundance of host plants. Importantly, southern populations of Brown Argus continue to use both plant species, especially in warm years when Geranium becomes common at the margins of rockrose habitat. This may maintain the ability of females to use both species of host plant, especially if Geranium host plants provide more productive larval hosts than rockrose, at least in years when summers are relatively warm and dry (Stewart et al. 2021, and in press). By contrast, use of rockrose at these sites may be maintained by cold and wet years, when eggs laid on these plants should show substantially lower mortality than those laid on Geranium plants (Stewart et al, in press). 
516 Rockrose dominated sites predominate at the existing northern range margin, which could be

517 a barrier to further expansion given the loss of rockrose use at new sites documented by

518 Buckley and Bridle (2014). However, two sites included here constitute new rockrose sites;

519 Forden is the northernmost site at the extreme northern range margin, and Barnack is the

520 southermost newly colonised site. There is some evidence for rapid local adaptation (10-12

521 generations) from Geranium to rockrose preference at Barnack (Bridle et al. 2014; Buckley

522 and Bridle 2014), driven either by evolution in situ, or re-colonisation from rockrose

523 favouring individuals from the range core. The latter is more likely, given high levels of gene-

524 flow found across the entire range $(\mathrm{Fst}=0.031)$, and the genetic similarity of new rockrose

525 sites despite the geographic distance between them (Figure 1, Figure 3). An alternative

526 explanation for the rockrose dominant site at the northern range edge (FOR) is that it is an

527 established A. agestis site which hadn't previously been detected. However, our assumption

528 that Forden is a newly-established A. agestis site is supported by our genetic evidence: FOR

529 clusters with the newly colonised sites in the fastStructure and fineRADstructure analyses and 530 the PCA.

The genomic basis of adaptation associated with range expansion

533 Using ddRAD markers allows us to investigate the genetic basis of adaptation associated with

534 climate-driven range expansion at many loci, an important extension to previous AFLP data

535 (Buckley et al 2012). In the first instance, it is remarkable that so few loci (and most with

536 relatively low Fst levels for the outliers) are associated with the host plant shift, given that the

537 plants are from different families. It could be that the shift in host plant is not as

538 phenotypically demanding as we imagine, with potentially substantial overlap in the butterfly

539 traits needed to sustain both interactions. Instead, the difference in microclimate between 
540 host-plants could be the main factor driving evolutionary change, demanding adaptation to

541 cope with different pathogen abundance associated with each plant, or shifts in egg

542 production or composition to alter desiccation or thermal resistance (see e.g. Stewart et al.

543 2021).

545 Alternatively, genomic variation already present and affecting host plant use in the

546 established populations may mean that only small changes in allele frequency across many

547 loci are needed to specialize on Geranium plants during expansion (see e.g. Pritchard et al.

548 2010). A. agestis is known to utilise Geraniaceae in the established part of the range where it

549 is available, although they prefer H. nummularium in choice experiments. In addition,

550 laboratory rearing of eggs (in warm conditions) is more successful on Geranium host plants

551 than on rockrose, which seem to present a less nutritious (albeit more climatically reliable)

552 host for larvae (Stewart et al. in press). We note also that we know nothing currently about

553 local adaptation in the northern populations of these host plants, or any evolutionary

554 responses that may have occurred in response to the novel invasion of Brown Argus into their

555 communities. It seems likely that alleles determining use of a given host plant in Brown

556 Argus will vary across its geographical range, possibly in response to plant local adaptation,

557 and in relation to local microclimate (Stewart et al, 2021), as has been suggested in the

558 Glanville Fritillary in Aaland (de Jong et al. 2018).

559

560 Two of the loci associated with host plant choice were located on the X-chromosome (Figure

561 4), which corresponds with evidence that the X-chromosome is important in Lepidopteran

562 speciation driven by host-plant differences (Sperling 1994; Prowell 1998; Janz 2019). Within

563 species variation in female oviposition choice has also associated with loci on the X- 
chromosome (e.g. in the comma buttefly, Nygren et al. 2006). Theoretically, genes on the Xchromosome could evolve faster than genes on autosomes, because that the recessive genes will be more available for selection to act on in the heterogametic sex (Charlesworth et al. 1987). It follows that a rapid change in female oviposition preference would be most successful if the genes were located on the X-chromosome. In contrast, a multi-species comparison of genes associated with host-plant preference in Lepidoptera found that loci were distributed throughout the genome, with a core set of genes located on the autosomes found across all butterfly/plant pairs (Nallu et al. 2018). A BLAST search of our loci found no overlap between our outlier loci and these candidate genes.

574 The four loci associated with both colonisation history and host-plant prevalence are particularly interesting because individuals colonising new sites during range expansions are often high dispersers. Increased individual movement (and extended searching) is also associated with Geranium host plant use (Bodsworth 2002). In addition, Bridle et al (2014)

578 provide evidence for the evolution of increased dispersal ability in A. agestis in newly-

579 colonised sites in the UK. However, a BLAST search of the candidate regions associated with 580 dominant host plant or colonisation history did not reveal anything of particular interest 581 (Table S1).

\section{Understanding the evolution of biotic interactions under climate change}

584 This study provides further evidence that (a) evolutionary responses have been necessary for 585 climatic shifts in the Brown Argus butterfly in the UK, and that (b) such evolution has 586 occurred in situ, and has involved shifts in polygenic traits, involving the creation and 587 establishment of novel genotypes during the range expansion, rather than the colonisation of 
pre-existing genotypes from elsewhere in the range. The Brown Argus example is highly instructive, given that rapid evolutionary responses are likely to be necessary for climate adaptation in many species and communities characterised by specialist biotic interactions,

591 and look likely to depend on the maintenance of gene flow across a species' geographical 592 range (Hoffmann et al. 2021). Increased environmental unpredictability as well as variation 593 are key in shaping the life history and behavioural strategies that evolve during adaptation to novel climates (Hoffmann \& Bridle 2021). In this context, the Brown Argus example is also important, given its rapid range expansion is likely to have reduced its capacity to cope with

596 increasingly unpredictable conditions in coming decades, by favouring specialisation on a

597 host plant that is only productive during clement years (Stewart et al, in press). We may 598 therefore expect that, due to evolutionary responses associated with range expansion, UK 599 Brown Argus populations in coming decades will be characterised by greater fluctuations in 600 population size, and loss of genetic variation in coming decades (O'Brien et al. in press), at 601 least until selection favours the re-establishment of rockrose preference at the edge of its 602 range.

603

\section{Acknowledgements}

605

606 MdJ was funded by a Marie Curie Postdoctoral Fellowship. AJvR was funded by a Swiss 607 National Science Foundation Early Postdoc Mobility Fellowship (P2ZHP2_178363). The 608 generation of genomic data was funded by a grant to JB and MdJ from the Biomolecular 609 Analysis Facility (NBAF) of the UK's Natural Environment Research Council (NERC). We 610 thank Roger Butlin and Chris Thomas for useful discussions and advice on sampling and data 
analysis, and to Natural England, the National Trust, individual landowners, and XXXXXXX

for permission to collect, and for assistance in the field.

\section{Data accessibility}

Sequence data will be submitted to the SRA on NCBI. Data and scripts used for each analysis

will be uploaded to Dryad.

\section{References}

Angert AL, Bontrager MG, Ågren J (2020) What Do We Really Know About Adaptation at Range Edges? Annual Review of Ecology, Evolution, and Systematics, 51, 341-361.

Asher J, Warren M, Fox R et al. (2001) The millennium atlas of butterflies in Britain and Ireland. Oxford University Press.

Van Belleghem SM, Vangestel C, De Wolf K et al. (2018) Evolution at two time frames: polymorphisms from an ancient singular divergence event fuel contemporary parallel evolution. PLoS genetics, 14, e1007796.

Bodsworth E (2002) Dispersal and behaviour of butterflies in response to their habitat. University of Leeds.

Bolger AM, Lohse M, Usadel B (2014) Trimmomatic: A flexible trimmer for Illumina sequence data. Bioinformatics, 30, 2114-2120.

Bridle JR, Buckley J, Bodsworth EJ, Thomas CD (2014) Evolution on the move: specialization on widespread resources associated with rapid range expansion in response to climate change. Proceedings of the Royal Society B: Biological Sciences, 281, 20131800.

Bridle JR, Kawata M, Butlin RK (2019) Local adaptation stops where ecological gradients steepen or are interrupted. Evolutionary Applications, 12, 1449-1462.

Bridle JR, Polechova J, Vines TH (2009) Patterns of biodiversity and limits to adaptation in time and space. In: Speciation and patterns of biodiversity (eds Butlin R., Bridle JR, Schluter D), pp. 77-101. Cambridge University Press, Cambridge, UK.

Bridle J, van Rensburg A (2020) Discovering the limits of ecological resilience. Science, 367, $626 \mathrm{LP}-627$.

Buckley J, Bridle JR (2014) Loss of adaptive variation during evolutionary responses to climate change. Ecology Letters, 17, 1316-1325.

Buckley J, Butlin RK, Bridle JR (2012) Evidence for evolutionary change associated with the recent range expansion of the British butterfly, Aricia agestis, in response to climate change. Molecular ecology, 21, 267-280.

Charlesworth B, Coyne JA, Barton NH (1987) The Relative Rates of Evolution of Sex Chromosomes and Autosomes. The American Naturalist, 130, 113-146.

Chen I-C, Hill JK, Ohlemüller R, Roy DB, Thomas CD (2011) Rapid Range Shifts of Species Associated with High Levels of Climate Warming. Science, 333, 1024-1026.

Cingolani P, Platts A, Wang LL et al. (2012) A program for annotating and predicting the effects of single nucleotide polymorphisms, SnpEff: SNPs in the genome of Drosophila 
melanogaster strain w1118; iso-2; iso-3. Fly, 6, 80-92.

Danecek P, Auton A, Abecasis G et al. (2011) The variant call format and VCFtools. Bioinformatics, 27, 2156-2158.

Eaton DAR (2014) PyRAD: Assembly of de novo RADseq loci for phylogenetic analyses. Bioinformatics, 30, 1844-1849.

Excoffier L, Dupanloup I, Huerta-Sánchez E, Sousa VC, Foll M (2013) Robust demographic inference from genomic and SNP data. PLoS genetics, 9, e1003905-e1003905.

Foll M, Gaggiotti O (2008) A genome-scan method to identify selected loci appropriate for both dominant and codominant markers: A Bayesian perspective. Genetics, 180, 977993.

Goudet J (2005) Hierfstat, a package for R to compute and test hierarchical F-statistics. Molecular Ecology Notes, 5, 184-186.

Hanski I, Breuker CJ, Schöps K, Setchfield R, Nieminen M (2002) Population history and life history influence the migration rate of female Glanville fritillary butterflies. Oikos, $\mathbf{9 8 ,}$ 87-97.

Heath J, Pollard E, Thomas JA (1984) Atlas of butterflies in Britain and Ireland. Viking.

Hill JK, Griffiths HM, Thomas CD (2011) Climate Change and Evolutionary Adaptations at Species' Range Margins. Annual Reviews in Entomology, 56, 143-159.

Hoffmann AA, Bridle J (2021) The dangers of irreversibility in an age of increased uncertainty: revisiting plasticity in invertebrates. Oikos.

Hoffmann AA, Miller AD, Weeks AR (2021) Genetic mixing for population management: From genetic rescue to provenancing. Evolutionary Applications, 14, 634-652.

Hoffmann A a, Sgrò CM (2011) Climate change and evolutionary adaptation. Nature, 470, 479-85.

Hoffmann AA, Sgrò CM (2018) Comparative studies of critical physiological limits and vulnerability to environmental extremes in small ectotherms: How much environmental control is needed? Integrative Zoology, 13, 355-371.

Jaenike J (1990) Host specialization in phytophagous insects. Annual Review of Ecology and Systematics, 21, 243-273.

Janz N (2019) Sex Linkage of Host Plant Use in Butterflies. In: Butterflies: Ecology and Evolution Taking Flight (eds Boggs CL, Watt WB, Ehrlich PR), pp. 229-240. University of Chicago Press.

Jombart T, Ahmed I, Calboli F et al. (2008) Package ' adegenet .' Bioinformatics Application Note, 24, 1403-1405.

Jones FC, Chan YF, Schmutz J et al. (2012) A Genome-wide SNP Genotyping Array Reveals Patterns of Global and Repeated Species-Pair Divergence in Sticklebacks. Current Biology, 22, 83-90.

Kautt AF, Kratochwil CF, Nater A et al. (2020) Contrasting signatures of genomic divergence during sympatric speciation. Nature, 588, 106-111.

Keightley PD, Pinharanda A, Ness RW et al. (2014) Estimation of the Spontaneous Mutation Rate in Heliconius melpomene. Molecular Biology and Evolution, 32, 239-243.

Kopp M, Matuszewski S (2014) Rapid evolution of quantitative traits: theoretical perspectives. Evolutionary Applications, 7, 169-191.

Li H (2011) A statistical framework for SNP calling, mutation discovery, association mapping and population genetical parameter estimation from sequencing data. Bioinformatics, 27, 2987-2993.

Li H (2013) Aligning sequence reads, clone sequences and assembly contigs with BWAMEM. bioRxiv, 00, 1-3. 
Li H, Handsaker B, Wysoker A et al. (2009) The Sequence Alignment/Map format and SAMtools. Bioinformatics, 25, 2078-2079.

Luu K, Bazin E, Blum MGB (2016) pcadapt: An R package to perform genome scans for selection based on principal component analysis. Molecular Ecology Resources, 33, 6777.

Malinsky M, Trucchi E, Lawson DJ, Falush D (2018) RADpainter and fineRADstructure : Population Inference from RADseq Data. Molecular Biology and Evolution, 35, 1284 1290.

Manichaikul A, Mychaleckyj JC, Rich SS et al. (2010) Robust relationship inference in genome-wide association studies. Bioinformatics, 26, 2867-2873.

Nadeau CP, Urban MC, Bridle JR (2017) Climates Past, Present, and Yet-to-Come Shape Climate Change Vulnerabilities. Trends in Ecology \& Evolution, 32, 786-800.

Nallu S, Hill JA, Don K et al. (2018) The molecular genetic basis of herbivory between butterflies and their host plants. Nature ecology \& evolution, 2, 1418-1427.

Nei M (1973) Analysis of Gene Diversity in Subdivided Populations. Proceedings of the National Academy of Sciences, 70, 3321-3323.

Nygren GH, Nylin S, Stefanescu C (2006) Genetics of host plant use and life history in the comma butterfly across Europe: varying modes of inheritance as a potential reproductive barrier. Journal of Evolutionary Biology, 19, 1882-1893.

Oksanen J, Blanchet FG, Kindt R et al. (2015) Vegan: community ecology package. R package vegan, vers. 2.2-1.

Paradis E (2010) pegas: an R package for population genetics with an integrated-modular approach. Bioinformatics, 26, 419-420.

Parmesan C (2006) Ecological and Evolutionary Responses to Recent Climate Change. Annual Review of Ecology, Evolution, and Systematics, 37, 637-669.

Pateman RM, Hill JK, Roy DB, Fox R, Thomas CD (2012) Temperature-Dependent Alterations in Host Use Drive Rapid Range Expansion in a Butterfly. Science, 336, 1028 LP - 1030.

Patterson M, Marschall T, Pisanti N et al. (2015) WhatsHap: weighted haplotype assembly for future-generation sequencing reads. Journal of Computational Biology, 22, 498-509.

Peterson BK, Weber JN, Kay EH, Fisher HS, Hoekstra HE (2012) Double digest RADseq: An inexpensive method for de novo SNP discovery and genotyping in model and nonmodel species. PloS one, 7.

Platts PJ, Mason SC, Palmer G et al. (2019) Habitat availability explains variation in climatedriven range shifts across multiple taxonomic groups. Scientific Reports, 9, 15039.

Polechová J, Barton NH (2015) Limits to adaptation along environmental gradients. Proceedings of the National Academy of Sciences of the United States of America, 112, 6401-6406.

Pritchard JK, Pickrell JK, Coop G (2010) The Genetics of Human Adaptation: Hard Sweeps, Soft Sweeps, and Polygenic Adaptation. Current Biology, 20, R208-R215.

Pritchard JK, Stephens M, Donnelly P (2000) Inference of population structure using multilocus genotype data. Genetics, 155, 945-959.

Prowell DP (1998) Endless forms: Species and speciation.

Purcell S, Neale B, Todd-Brown K et al. (2007) PLINK: A tool set for whole-genome association and population-based linkage analyses. American Journal of Human Genetics, 81, 559-575.

Raj A, Stephens M, Pritchard JK (2014) FastSTRUCTURE: Variational inference of population structure in large SNP data sets. Genetics, 197, 573-589. 
Sperling FAH (1994) Sex-linked genes and species differences in Lepidoptera. The Canadian Entomologist, 126, 807-818.

Stålhandske S, Olofsson M, Gotthard K et al. (2016) Phenological matching rather than genetic variation in host preference underlies geographical variation in host plants used by orange tip butterflies. Biological Journal of the Linnean Society, 119, 1060-1067.

Stewart JE, Maclean IMD, Edney AJ, Bridle J, Wilson RJ (2021) Microclimate and resource quality determine resource use in a range-expanding herbivore. Biology Letters, 17 , 20210175.

The UniProt Consortium (2021) UniProt: the universal protein knowledgebase in 2021. Nucleic Acids Research, 49, D480-D489.

Thomas CD, Bodsworth EJ, Wilson RJ et al. (2001) Article : Ecological and evolutionary processes at expanding range margins. Nature, 411, 577-581.

Tolman T (1997) Butterflies of Britain and Europe. Harpercollins Pub Limited.

Warren MS, Hill JK, Thomas JA et al. (2001) Rapid responses of British butterflies to opposing forces of climate and habitat change. Nature, 414, 65-69.

\section{Author contributions:}

- MdJ devised the study, secured ERC and NBAF funding, conducted the sampling and molecular lab work for the population genomics dataset, and data interpretation, and helped write the manuscript.

- AJvR designed and conducted the bioinformatic and genomic analysis and interpretation, secured NBAF funding, and helped write the manuscript.

- SW designed and conducted bioinformatic analysis and created the draft genome assembly.

- CJY conducted the molecular lab work for the draft genome assembly

- JRB devised the study, hosted MdJ and assisted with study and sampling design, fieldwork, data analysis and interpretation, and helped write the manuscript (with contributions from all authors)

- $\mathrm{MB}$ assisted with genomic analysis and data interpretation 
bioRxiv preprint doi: https://doi.org/10.1101/2022.02.07.479435; this version posted February $9,2022$. The copyright holder for this

preprint (which was not certified by peer review) is the author/funder, who has granted bioRxiv a license to display the preprint in perpetuity. It is made available under aCC-BY-NC-ND 4.0 International license.

- CJ co-hosted MdJ and assisted with sampling design, genomic analysis and data interpretation. 


\section{Tables and Figures}

\section{Table 1: Sample sites and locations}

Characteristics of the nine $A$. agestis sites sampled across the UK, ordered from south to north. The number of individuals (N) included in the final analysed dataset is shown, with the number of sequenced individuals in brackets. Sites were defined as established (occupied since 1970-1982) or new (occupied since 1995-1997), and as Geraniaceae or Cistaceae based on the dominant host plant at that site. We report two measures of genetic diversity; nucleotide diversity $(\pi)$ and expected heterozygosity (Hexp; see Figure S1) as the mean and standard deviation (SD) estimated across all neutral SNPs. Nei's F ST was estimated for between all nine populations (Overall), and between the Colonisation History (Est vs New) and Host Plant (Cis vs Ger) groups, as well as between populations within the Established (within Est) and New sites (within New). HOD was excluded from the within Est comparison given its geographic distance from the established South.

\begin{tabular}{|c|c|c|c|c|c|c|c|c|c|c|}
\hline Site & Population & $\mathbf{N}$ & Lat & Long & Colonisation History & Host Plant & $\pi(\mathrm{SD})$ & Hexp (SD) & Fst & \\
\hline$\overline{\text { Beacon Hill }}$ & $\mathrm{BCH}$ & $38(39)$ & 50.998 & -1.136 & established & Cistaceae & $0.0012(0.0011)$ & $0.178(0.16)$ & Overall & 0.031 \\
\hline Lydden & LYD & $20(23)$ & 51.154 & 1.269 & established & Cistaceae & $0.0011(0.0009)$ & $0.176(0.17)$ & Est vs New & 0.026 \\
\hline Swyncombe & SWD & $38(40)$ & 51.618 & -1.039 & established & Cistaceae & $0.0011(0.0010)$ & $0.178(0.16)$ & Cis vs Ger & 0.018 \\
\hline Barnack & BAR & $28(30)$ & 52.629 & -0.414 & new & Cistaceae & $0.0011(0.0010)$ & $0.177(0.17)$ & within Est* & 0.016 \\
\hline Holme Dunes & HOD & $29(34)$ & 52.973 & 0.543 & established & Geraniaceae & $0.0011(0.0010)$ & $0.178(0.16)$ & within New & 0.014 \\
\hline Moor Farm & MOF & $25(26)$ & 53.156 & -0.169 & new & Geraniaceae & $0.0012(0.0011)$ & $0.173(0.17)$ & & \\
\hline Whisby & WIS & $38(45)$ & 53.190 & -0.640 & new & Geraniaceae & $0.0012(0.0011)$ & $0.173(0.17)$ & & \\
\hline Brockadale & BRO & $15(19)$ & 53.645 & -1.219 & new & Geraniaceae & $0.0011(0.0010)$ & $0.171(0.18)$ & & \\
\hline Fordon & FOR & $20(20)$ & 54.162 & -0.394 & new & Cistaceae & $0.0009(0.0008)$ & $0.174(0.18)$ & & \\
\hline
\end{tabular}

*excludes HOD 


\section{Table 2 Redundancy Analysis}

Partitioning of genetic variance in each geographic transect using RDA and partial RDA analyses. The column "Partitioned variance" shows the total variance of the genetic data (Total variance), the proportion of variance that could be explained by the full RDA model which includes HostPlant, Colonisation History, Longitude and Latitude as explanatory variables (Basic Model), and the proportion of total variance explained by the partial RDA in each case. The column "Proportion constrained" shows the variation explained by each model relative to the total explainable variance. The fit $\left(\mathrm{R}^{2} \mathrm{adj}\right)$ and significance $(\mathrm{P})$ of each model is shown, and with significant $\mathrm{p}$-values shown in bold.

\section{Basic Model}

\begin{tabular}{|c|c|c|c|c|}
\hline Genetic variation & Partitioned variance & Proportion constrained & R2adj & $\mathbf{P}$ \\
\hline Total variance & 325.10 & & & \\
\hline Full model (constrained variance) & 214.15 & 1.00 & 0.66 & $0.001 * * *$ \\
\hline Host plant only (Host Plant / ColHist + Geog) & 32.20 & 0.10 & 0.02 & 0.39 \\
\hline Colonisation history only (ColHist / Geog+HostPlant) & 43.99 & 0.14 & 0.08 & 0.15 \\
\hline Geog only (Lat + Long / HostPlant + ColHist) & 93.73 & 0.29 & 0.16 & 0.06 \\
\hline
\end{tabular}

\section{Table 3 Comparison of demographic models}

Three demographic models (Figure 2) considering a) asymmetric migration between populations, and b) no migration after population divergence were simulated using FastSimCoal2. We report the $\log$ likelihood $\left(\log _{10} \mathrm{~L}\right)$, number of parameters estimated (k), Akaike Information Criterion (AIC), rescaled AIC ( $\triangle \mathrm{AIC}$ ), and weighting (wi) for each model. The best-supported model is highlighted in bold.

\begin{tabular}{rllllll} 
& Model & Log10L & k & AIC & deltaAIC & wi \\
\hline 1a & -19917.8 & 15 & 91752.86 & 45.41 & 0.00 \\
$1 \mathrm{~b}$ & -19944.37 & 9 & 91863.20 & 155.75 & 0.00 \\
& 2a & -20007.05 & 15 & 92163.88 & 456.43 & 0.00 \\
& 2b & -20019.58 & 9 & 92209.59 & 502.14 & 0.00 \\
& 3a & $\mathbf{- 1 9 9 0 7 . 0 7}$ & $\mathbf{1 7}$ & $\mathbf{9 1 7 0 7 . 4 5}$ & $\mathbf{0 . 0 0}$ & $\mathbf{1 . 0 0}$ \\
811 & 3b & -19933.18 & 11 & 91815.67 & 108.22 & 0.00 \\
\hline
\end{tabular}




\section{Table 4. Demographic parameters estimated from the best-supported coalescent model: Model3a}

Point estimates were obtained from the run with the highest composite maximum likelihood. Upper and lower bounds were estimated from 100 bootstrap replicates of the model using these point estimates to construct the simulated site frequency spectrum. The parameter names are defined Figure 2 (model 3a), and priors are shown in the online supplementary material (Table S4).

\begin{tabular}{lllll}
\hline Parameter & Estimate & $\begin{array}{l}\text { Lower } \\
\text { Bound }\end{array}$ & $\begin{array}{l}\text { Upper } \\
\text { Bound }\end{array}$ & Category \\
\hline ANCSIZE & 544351 & 30800 & 1119249 & $\begin{array}{l}\text { Population size } \\
\text { NNEW }\end{array}$ \\
15562 & 5560 & 44915 & $\begin{array}{l}\text { Population size } \\
\text { Population size }\end{array}$ \\
NSOUTH & $86206^{*}$ & & & Population size \\
NHOD & 16659 & 8691 & 65541 & Time \\
TADMIX & 9511 & 3680 & 13034 & Time (complex parameter) \\
TPLUSDIV & 18372 & 4564 & 19874 & Migration \\
MIG01 & $2.31 \mathrm{E}-04$ & $5.71 \mathrm{E}-10$ & $6.56 \mathrm{E}-04$ & Migration \\
MIG10 & $1.57 \mathrm{E}-08$ & $3.82 \mathrm{E}-12$ & $7.31 \mathrm{E}-05$ & Migration \\
MIG02 & $4.78 \mathrm{E}-07$ & $3.67 \mathrm{E}-11$ & $3.30 \mathrm{E}-04$ & Migration \\
MIG20 & $2.35 \mathrm{E}-05$ & $3.51 \mathrm{E}-10$ & $4.44 \mathrm{E}-04$ & Migration \\
MIG12 & $1.45 \mathrm{E}-04$ & $3.30 \mathrm{E}-11$ & $7.67 \mathrm{E}-05$ & Migration \\
MIG21 & $8.97 \mathrm{E}-05$ & $1.19 \mathrm{E}-11$ & $3.22 \mathrm{E}-04$ & Mutation rate \\
MUTRATE & $3.53 \mathrm{E}-09$ & $3.26 \mathrm{E}-09$ & $5.47 \mathrm{E}-09$ & Relative change in population size \\
RESIZE1 & 5.54 & 1.92 & 15.50 & Relative change in population size \\
RESIZE2 & 1.07 & 0.56 & 6.27 & Migration \\
MIGTOM & 0.56 & 0.11 & 0.95 & Relative change in population size \\
RESIZE3 & 6.31 & 0.36 & 12.98 & Time \\
TDIV1 & 27883 & 11227 & 29246 &
\end{tabular}




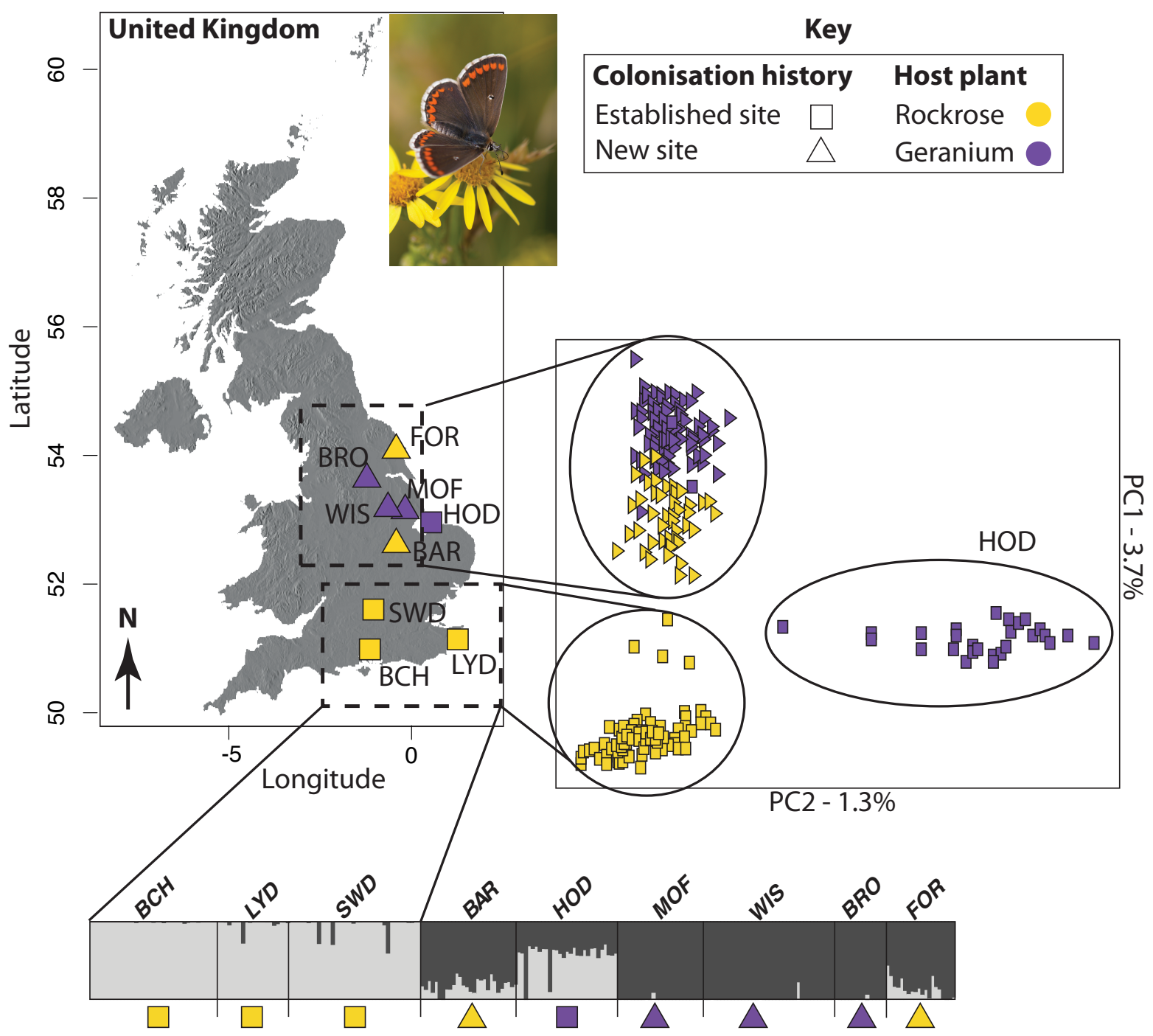

822

823

824

825

826

827

828

829

830

831

832

833

834

835

836

\section{Figure 1 Population structure}

Population structure of $A$. agestis sampled across their latitudinal range in the UK.

Colonisation history and dominant host plant at each site is shown with shapes and colours.

A) The geographic locations of sampled sites. B) Partitioning of genetic variance on a PCA. Note that the graph has been rotated to reflect the latitudinal gradient. PC1 explained $3.6 \%$ of the genetic variance and separates the northern and southern populations. PC2 explained 1.3 $\%$ of the variation and separates HOD from the rest of the samples. C) The proportion assignment of each individual to one of two genetic clusters as estimated by fastStructure. Each vertical bar represents an individual, with populations ordered from South to North. Photo credit: Callum Macgregor. 

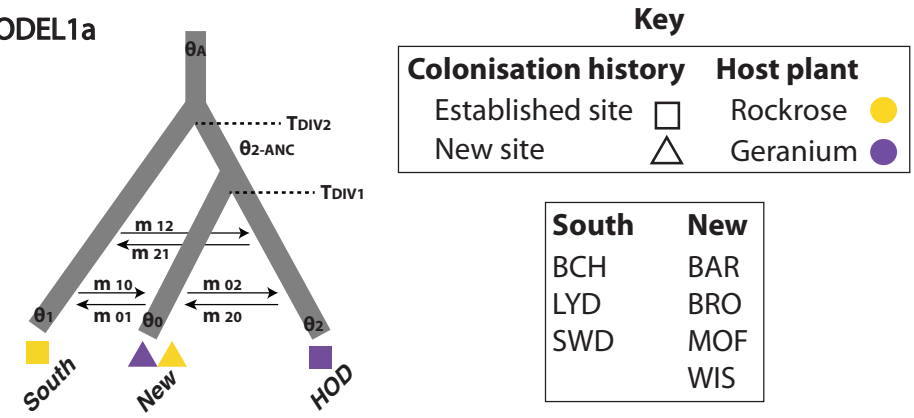

MODEL2a
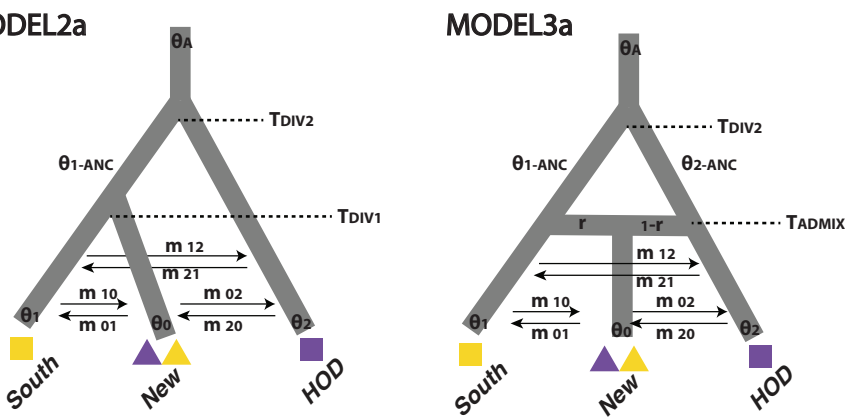

\section{Figure 2 Demographic models tested with fastSim Coal2}

839 Three demographic models were compared using FastSimCoal2: Model 1) Colonisation of the new sites exclusively from HOD followed by gene flow between all populations, Model 2) Colonisation of new sites exclusively from the South followed by gene flow between all populations, Model 3) Secondary contact and subsequent colonisation of the new sites by admixed populations from HOD and the South. All models were tested with two different migration matrices: a) assymetric gene-flow after divergence, b) no gene flow. Parameters included in the model were mutation-scaled effective population size $(\theta)$, migration rates per generation $(m)$, the time of divergence $\left(\mathrm{T}_{\mathrm{DIV}}\right)$ or admixture $\left(\mathrm{T}_{\mathrm{ADM}}\right)$ between populations, and the proportion of the source population transferred to the sink population $(r)$. 


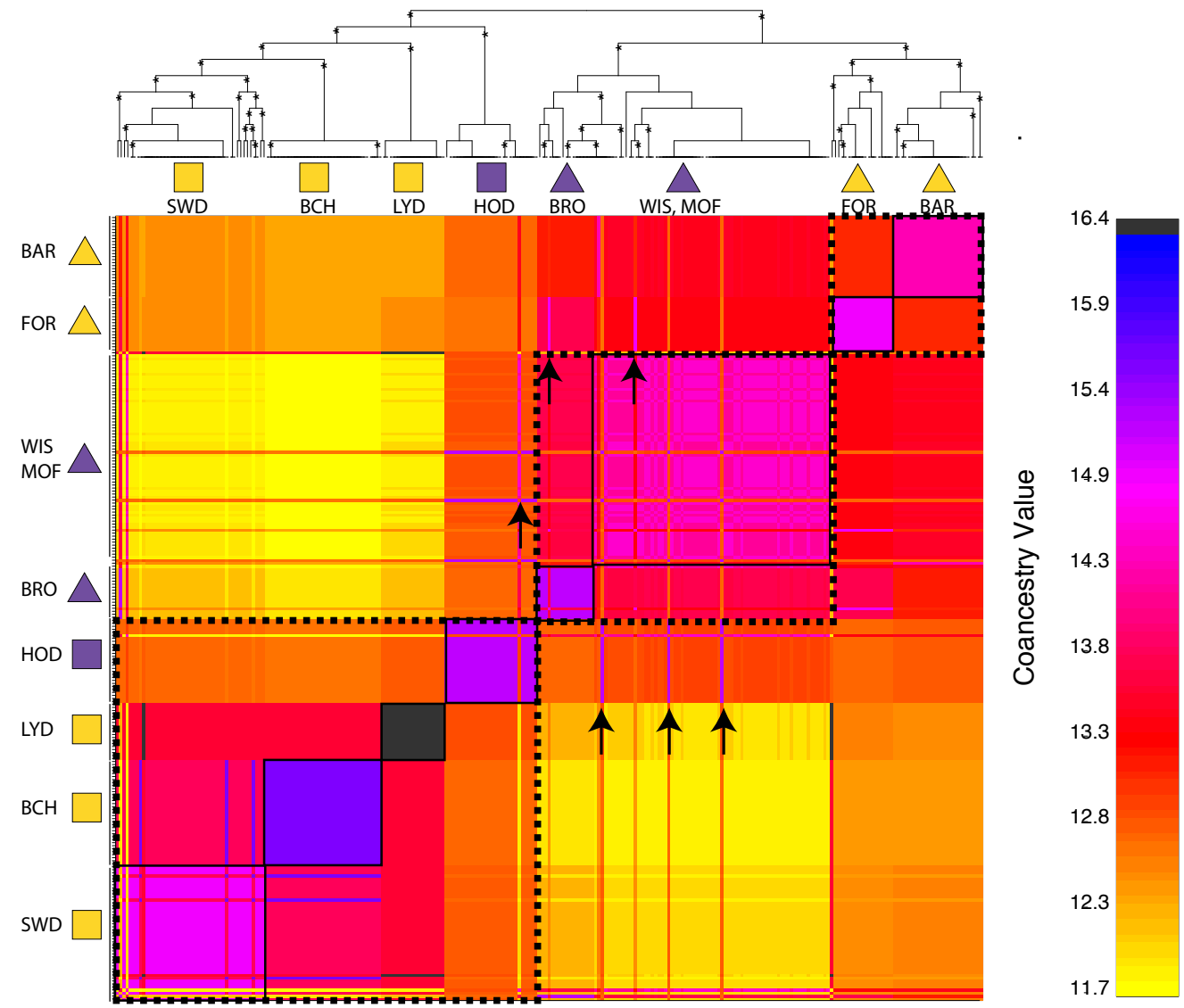

\section{Figure 3 fineRADstructure}

Individual coancestry matrix estimated with fineRADstructure and clustered by population using RADpainter. The level of co-ancestry is indicated with colour (high=black/blue; low=yellow) as shown by the colour bar to the right. The maximum a posteriori (MAP) tree shows the inferred ancestry of each individual. Posterior probability branch support above 0.85 is indicated with an asterisk. The inferred co-ancestry groups largely correspond to geographic populations, although there is evidence of haplotypes moving from the new Rockrose sites (BAR and FOR) to the new Geranium sites (WIS, MOF and BRO), as well between the new Geranium sites and HOD. These are indicated with arrows. Hierarchical structure in the data is shown with solid and dashed lines around grouped populations. 


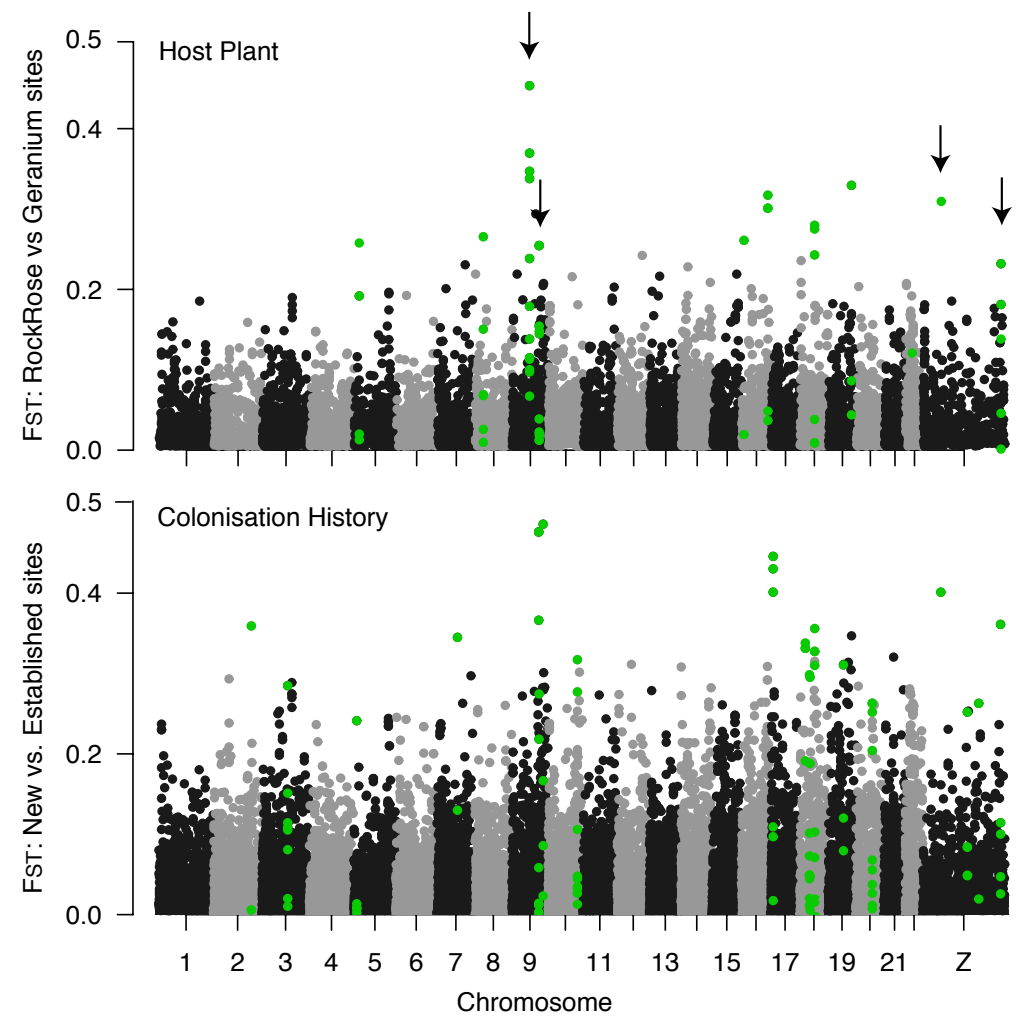

Figure 4 Manhattan plot

The per locus distribution of $\mathrm{F}_{\mathrm{ST}}$ across the genome between populations defined by A) Host Plant preference, and B) Colonisation history. Outlier loci are coloured green. Four outlier loci, indicated by arrows, were associated with both host plant choice and colonisation history. 

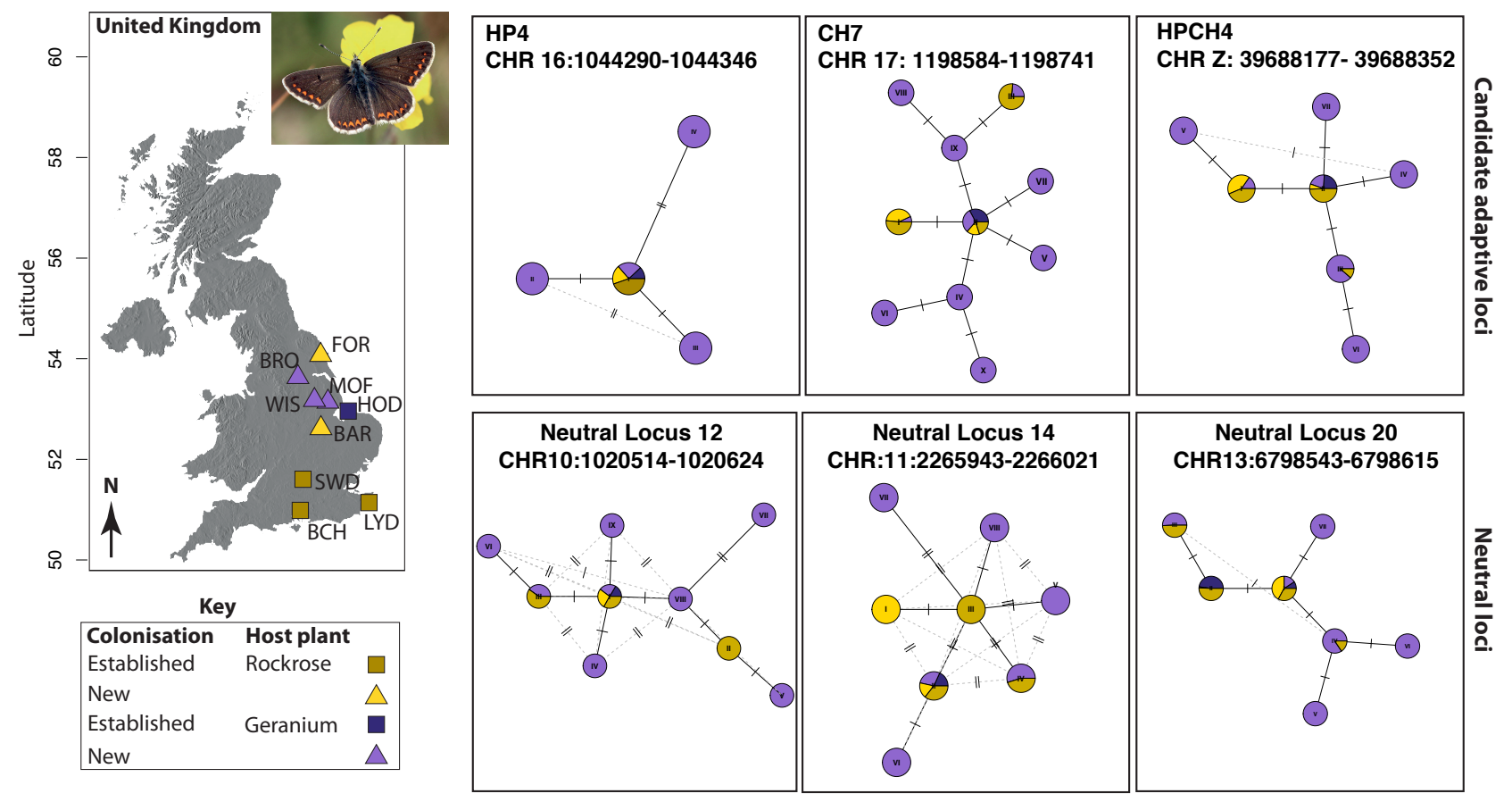

\section{Figure 5 Haplotype network}

Haplotype networks of a representative subset of the Host Plant (HP) outlier, Colonisation History $(\mathrm{CH})$ outlier and Neutral loci. The remaining loci showed the same overall pattern and are shown in Figure S2. A genomic location of each haplotype is shown in Table S2, and haplotype frequencies in Table S3. Light yellow and light purple represent the newly established populations, while dark yellow represents the established South and dark purple represents HOD, the potential source of Geraniaceae adaptive haplotypes. The star-like configuration of haplotypes is indicative of a recent expansion, where haplotypes found exclusively in the new sites radiate from more common haplotypes found in the established range. If the adaptive haplotypes were introduced from HOD, we would expect the light purple haplotypes to radiate from dark purple haplotypes. Instead, the adaptive haplotypes originate either from the established South, or from both the established South and from HOD. 
bioRxiv preprint doi: https://doi.org/10.1101/2022.02.07.479435; this version posted February 9, 2022. The copyright holder for this preprint (which was not certified by peer review) is the author/funder, who has granted bioRxiv a license to display the preprint in perpetuity. It is made available under aCC-BY-NC-ND 4.0 International license. 